\title{
The image jets modeling of gravitationally lensed sources
}

\section{Tatiana Larchenkova ${ }^{1}$, Alexander Lutovinov ${ }^{2}$ and Natalya Lyskova ${ }^{3}$}

${ }^{1}$ ASC of P. N. Lebedev Physical Institute, Profsoyuznaya str. 84/32, Moscow 117997, Russia email: tanya@lukash.asc.rssi.ru

${ }^{2}$ Space Research Institute, Profsoyuznaya str. 84/32, Moscow 117997, Russia

${ }^{3}$ Moscow Physical-Technical Institute, Institutskyi per., 9, Dolgoprudnyi 141700, Russia

\begin{abstract}
The jets image modelling of gravitationally lensed sources have been performed. Several basic models of the lens mass distribution were considered, in particular, a singular isothermal ellipsoid, an isothermal ellipsoid with the core, different multi-components models with the galactic disk, halo and bulge. The obtained jet images were compared as with each other as with results of observations. A significant dependence of the Hubble constant on the model parameters was revealed for B0218+357, when the circular structure was took into account.
\end{abstract}

Keywords. jets, gravitational lensing, Hubble constant, B0218+357

It is known, that due to the gravitational lensing of a compact object with a relativistic jet the multiple images of the object itself and of the jet can arise. Such gravitationally lensing systems are observed and the most bright from them are PKS 1830-211 (Nair et al., 1993) and B0218+357 (Patnaik et al., 1993). The modelling of images of such sources and study their temporal behavior are began actual due to forthcoming launches of cosmic radiointerferometers.

For the case of the lensing on the spiral galaxy we considered several multicomponents models: Model I - disk and softened halo, approximated by the isothermal ellipsoid located in the singular isothermal halo of a dark matter; Model II - disk, approximated by the Kuzmin disk (Kuzmin, 1956) in the isothermal halo; Model III - disk and bulge, approximated by the Kuzmin disk, in the isothermal halo (Keeton \& Kochanek 1998).
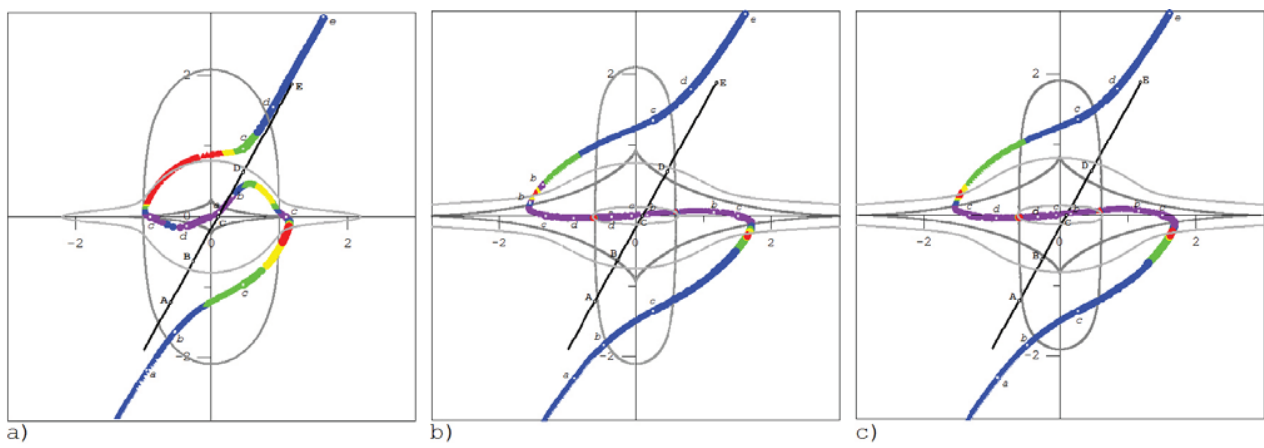

Figure 1. Mapping of a gravitationally lensed jet for three models (I - a, II - b, III - c). Designations are: gray - caustics, light-gray - critical curves, black line - jet. Jets images depending on the magnification $M$ are shown by magenta $(0<|M|<1)$, blue $(1<|M|<3)$, green $(3<|M|<7)$, yellow $(7<|M|<10)$ and red $(|M|>10)$. The lower case letters on the jet images correspond to the same capital letters on the jet itself (for details see Larchenkova et al. 2011). 


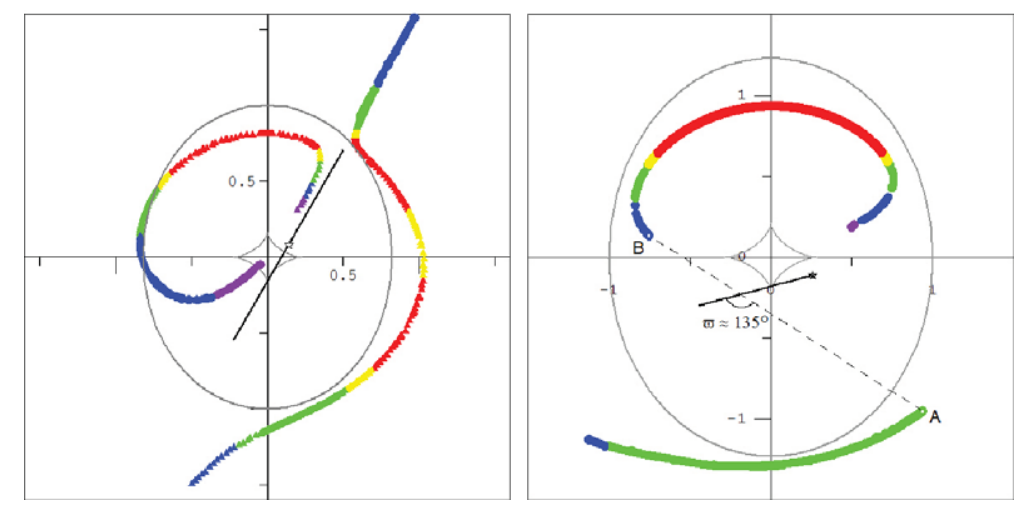

Figure 2. (left) The nearly circular image of the lensed jet in the Model I with parameters: $q_{3 d}=0.05, a_{d}=1.0$, jet slope 60 , inclination of all components $i=30$. (right) Results of the B0218+357 jet modelling with the Model I. sThe jet is directed at the angle of $\varpi \simeq 135^{\circ}$ to the line connected images $\mathrm{A}$ and $\mathrm{B}$, and cross the tangential caustic near its bottom cusp.

Depending on the disk axes ratio $q_{3 d}$, its characteristics size $a_{d}$ and jet parameters, the different geometry of caustics and jet images was obtained. Some typical results are presented in Fig. 1 for different lens models. It is clearly seen that the inclusion of the low-mass bulge (similar to the Milky Way one) does not change significantly the picture of the jet lensing (see Fig. 1 b, c).

There is a general question: is it possible to obtain a circular-like structure by the jet lensing? The one of answers is that: to obtain a circular-like structure it is necessary to cross the tangential caustics by the jet near tangentially to their cusps. An example of the appearance of such a structure are shown in Fig. 2 (left).

The system B0218+357 is a very interesting and important for astrophysical and cosmological investigations due to several reasons: the presence of a large scale jet and the circular-like structure in radio images; the time delay between images of the compact core, which was measured with the high accuracy; the significant distance from other extragalactic objects. For this system there were obtained several set of parameters of model I and II reproducing results of observations. An example of the mutual arrangement of the lens, source, jet and circular-like structure is presented in Fig. 2 (right). In a combination with the measured time delay the model parameters can give us estimations of the Hubble constant. It was revealed that the considered models lead to a wide variety of its value $H \simeq 35-90 \mathrm{~km} \mathrm{~s}^{-1} \mathrm{Mpc}^{-1}$ (for details see Larchenkova et al. 2011).

This work was supported by the program of Russian Academy of Sciences "Origin, Structure and Evolution of Objects in the Universe" and government contract P1336.

\section{References}

Keeton, C. R. \& Kochanek, C. S., 1998, ApJ, 495, 157

Kuzmin, G., 1956, Sov. Astron, 33, 27

Larchenkova, T., Lutovinov, A., \& Lyskova, N. 2011, Astron. Letters, in press

Nair, S., Narasimha, D., \& Rao, A. P., 1993, ApJ, 407, 46

Patnaik, A. et al., 1993, MNRAS, 261, 435 